\title{
The consumer's demand functions defined to study contingent consumption plans
}

\section{Summarized probability distributions: a mathematical application to contingent consumption choices}

\author{
Pierpaolo Angelini $^{2} \cdot$ Fabrizio Maturo $^{1}$ (D)
}

Accepted: 18 May 2021 / Published online: 24 May 2021

(c) The Author(s) 2021

\begin{abstract}
Given two probability distributions expressing returns on two single risky assets of a portfolio, we innovatively define two consumer's demand functions connected with two contingent consumption plans. This thing is possible whenever we coherently summarize every probability distribution being chosen by the consumer. Since prevision choices are consumption choices being made by the consumer inside of a metric space, we show that prevision choices can be studied by means of the standard economic model of consumer behavior. Such a model implies that we consider all coherent previsions of a joint distribution. They are decomposed inside of a metric space. Such a space coincides with the consumer's consumption space. In this paper, we do not consider a joint distribution only. It follows that we innovatively define a stand-alone and double risky asset. Different summary measures of it characterizing consumption choices being made by the consumer can then be studied inside of a linear space over $\mathbb{R}$. We show that it is possible to obtain different summary measures of probability distributions by using two different quadratic metrics. In this paper, our results are based on a particular approach to the origin of the variability of probability distributions. We realize that it is not standardized, but it always depends on the state of information and knowledge of the consumer.
\end{abstract}

Keywords 2-parallelepiped $\cdot \alpha$-product $\cdot \alpha$-norm $\cdot$ Antisymmetric tensor $\cdot$ Non-linear metric $\cdot$ Linear metric

Mathematics Subject Classification $60 \mathrm{~A} 05 \cdot 60 \mathrm{~B} 05 \cdot 91 \mathrm{~B} 24 \cdot 91 \mathrm{~B} 16 \cdot 91 \mathrm{~B} 06 \cdot 91 \mathrm{~B} 08$

Fabrizio Maturo

fabrizio.maturo@unicampania.it

Pierpaolo Angelini

pier.angelini@uniroma1.it

1 Dipartimento di Matematica e Fisica, Università degli Studi della Campania "L. Vanvitelli", Viale A. Lincoln, 5, 81100 Caserta, Italy

2 Dipartimento di Scienze Statistiche, Università "La Sapienza”, Piazzale A. Moro, 5, 00185 Roma, Italy 


\section{Introduction}

In this paper, an event is a logical entity (proposition) expressed by a real number and susceptible of taking two values only, $1=$ true and $0=$ false, as final answers characterizing the logic of certainty. An event can be certain or impossible, in a specific state of information and knowledge for the consumer, whenever its result is known with certainty and it is respectively true or false. An event is conversely possible whenever its result is not a priori known to her (de Finetti (1981)).

Let $X$ be a random gain whose true value is unknown for the consumer evaluating, but it is well-determined in itself (see also Gilio and Sanfilippo (2014)). Let $I(X)=\left\{x^{1}, x^{2}, \ldots, x^{m}\right\}$ be the set of all possible monetary values of $X$, where it turns out to be $x^{1}<x^{2}<\ldots<x^{m}$ without loss of generality. Such a set identifies an $m$-dimensional consumption vector denoted by

$$
\left(x^{1}, x^{2}, \ldots, x^{m}\right) \text {. }
$$

It expresses all possible quantitative states of nature of a contingent consumption plan.

Uncertainty about a state of nature of a contingent consumption plan has to be interpreted in a personalistic sense within this context, so it depends on a lack of information of the consumer evaluating (see also Capotorti et al. (2014)). Uncertainty about a state of nature of a contingent consumption plan consequently ceases only when she receives certain information about it (see also Machina (1987); de Finetti (1982b); Hoskova-Mayerova and Maturo (2018); Hošková-Mayerová et al. (2017); de Finetti (1989); Maturo (2018a, 2018b)).

Probability is associated with a state of nature of a contingent consumption plan. It always depends on the state of information and knowledge of the consumer evaluating (see also Coletti et al. (2016)). In this paper, we think of probability as being a mass. It is preferable to think of probability as being a mass whenever we are interested in distinguishing between the representation of the logical situation and the introduction of whatever coherent evaluation of probability the consumer wants to make (see also Viscusi and Evans (2006)). Probability is always a non-negative and additive function whose unit value is taken on the whole space of states of nature of a contingent consumption plan (see also Cassese et al. (2020)). Hence, axiomatic probability theory is satisfied. Nevertheless, the notion of probability associated with a state of nature of a contingent consumption plan is not undefined within this context. It is the degree of belief in the occurrence of a state of nature of a contingent consumption plan attributed by a given consumer at a given instant and with a given set of information and knowledge.

\subsection{A contingent consumption plan}

A function defined on the set of all possible quantitative states of nature of a contingent consumption plan coincides with $X$ (see also Berti et al. (2001)). Its domain and codomain coincide. They are expressed by $I(X)$. One and only one of all possible quantitative states of nature of a contingent consumption plan identifying $I(X)$ will be true at the right time (see also Berti and Rigo (2002)). We consider the finest possible partition into atomic (elementary) events. We say this thing because they are not further subdivisible for the purposes of the problem under consideration (with an event, but not with a set, it is always possible to 
continue the subdivision, even though it is convenient to stop as soon as the subdivision is good enough for the study in progress). That alternative which will turn out to be verified a posteriori is nothing but a random point contained in $I(X)$. It expresses everything there is to be said (Nunke and Savage (1952)).

Each random gain can coherently be assigned a distribution of probability as an expression of the attitude of the consumer under consideration (see also Schmeidler (1989)). It follows that each consumer is faced with $m$ masses denoted by $p_{1}, p_{2}, \ldots, p_{m}$ such that it is possible to write $p_{1}+p_{2}+\ldots+p_{m}=1$. They are located on $m$ real numbers denoted by $x^{1}, x^{2}, \ldots, x^{m}$ (see also Angelini and Maturo (2020)).

Each single state of nature of a contingent consumption plan could uniquely be expressed by infinite real numbers, so we could also write

$$
\left\{x^{1}+a, x^{2}+a, \ldots, x^{m}+a\right\},
$$

where $a \in \mathbb{R}$ is an arbitrary constant, to denote the set of all possible states of nature of a contingent consumption plan. We consider infinite translations in this way. It is possible to consider different quantities from a geometric point of view. They are nevertheless the same quantity from a randomness point of view because states of nature and probabilities associated with them do not change (see also Pfanzagl (1967)). We say that a change of origin is inessential from a randomness point of view (see also Dinçer et al. (2020)).

\subsection{Contravariant and covariant indices of a probability distribution associated with a contingent consumption plan}

Let $E_{i}, i=1, \ldots, m$, be the generic state of nature of a contingent consumption plan.

We establish the following

Definition 1 Let $X$ be a random gain. We say that $x^{1}$ is the return on $X$ if $E_{1}$ occurs with probability denoted by $p_{1}, \ldots, x^{m}$ is the return on $X$ if $E_{m}$ occurs with probability denoted by $p_{m}$. We note that $x^{1}$ is the wealth that $X$ yields and that can be spent by the consumer if $E_{1}$ occurs with probability denoted by $p_{1}, \ldots, x^{m}$ is the wealth that $X$ yields and that can be spent by the consumer if $E_{m}$ occurs with probability denoted by $p_{m}$.

Thus, we write

$$
\left(x^{1}, p_{1}\right),\left(x^{2}, p_{2}\right), \ldots,\left(x^{m}, p_{m}\right)
$$

in order to identify a discrete probability distribution of $X$.

A probability distribution is expressed by using contravariant indices together with covariant ones. We wish to distinguish possibility (studied by the logic of certainty) from probability (studied by the logic of uncertainty or probability theory) in this way (see also Anscombe and Aumann (1963)). We wish to distinguish what is true or false without gradations (there are no degrees of possibility) from what is more or less probable in opinion of a given consumer. We use contravariant indices to identify the possible monetary values of $X$. We use covariant indices to denote the corresponding probabilities that are assigned to them. On the other hand, contravariant and covariant components of a same vector are equal whenever we use an orthonormal basis of the real linear space under consideration (Pompilj (1957)). 


\section{A multivariate approach to the consumer's demand functions connected with contingent consumption plans}

Let ${ }_{1} X$ and ${ }_{2} X$ be two linearly independent random gains (risky assets), where the number of the possible monetary values of each of them is equal to $m$ without loss of generality. We deal with two contingent consumption plans, where contingent means depending on something not yet sure. Let $I\left({ }_{1} X\right)=\left\{{ }_{(1)} x^{1}, \ldots,{ }_{(1)} x^{m}\right\}$ and $I\left({ }_{2} X\right)=\left\{{ }_{(2)} x^{1}, \ldots,{ }_{(2)} x^{m}\right\}$ be the sets of possible values for ${ }_{1} X$ and ${ }_{2} X$. Such sets contain the contravariant components of two $m$-dimensional and linearly independent vectors. The possible quantitative states of nature of two contingent consumption plans are therefore found on the two mutually orthogonal axes of a two-dimensional Cartesian coordinate system on which an origin, a same unit of length and an orientation are established (von Neumann (1936)). We say that the two contingent consumption plans under consideration are logically independent. It follows that all the $\mathrm{m}^{2}$ possible monetary values of two random gains jointly considered belong to a subset of a two-dimensional Cartesian coordinate system (see also Regazzini (1985)). On the other hand, we can always write ${ }_{1} X={ }_{1} Y-{ }_{1} Z$, where we have ${ }_{1} Y={ }_{1} X\left({ }_{1} X \geq 0\right)$ and ${ }_{1} Z=-{ }_{1} X\left({ }_{1} X \leq 0\right)$. The term gain has intrinsically to be understood in an algebraic sense, so a loss is a negative gain. The possible monetary values of ${ }_{1} Y$ and ${ }_{1} Z$ are always non-negative because it turns out to be ${ }_{1} Y={ }_{1} X$, if ${ }_{1} X>0$ and zero otherwise, as well as ${ }_{1} Z=-{ }_{1} X$, if ${ }_{1} X<0$ and zero otherwise. It is possible to suppose that the number of the possible monetary values of ${ }_{1} Y$ is the same of the one of the possible monetary values of ${ }_{1} Z$. Also, we suppose that zero (whose probability is equal to 0 , so we have $0 \cdot 0=0$ ) always separates them. The same is true by considering ${ }_{2} X={ }_{2} Y-{ }_{2} Z$. This thing is in general valid. This is because if $a$ is a real number lying between $a^{\prime}$ and $a^{\prime \prime}$ then we observe that $a_{1} X$ lies between $a^{\prime}{ }_{1} X$ and $a^{\prime \prime}{ }_{1} X$ when and only when $I\left({ }_{1} X\right)$ contains all non-negative numbers. Differently, all of this is not true. Since it is possible to write

$$
\mathbf{P}\left(a_{1} X\right)=a \mathbf{P}\left({ }_{1} X\right)
$$

for every real number denoted by $a$, we observe that if $a$ lies between $a^{\prime}$ and $a^{\prime \prime}$ then $\mathbf{P}\left(a_{1} X\right)$ lies between $\mathbf{P}\left(a^{\prime}{ }_{1} X\right)$ and $\mathbf{P}\left(a^{\prime \prime}{ }_{1} X\right)$, where $\mathbf{P}$ is obtained by using the same probabilities with regard to $I\left({ }_{1} X\right)$ containing all non-negative numbers. The same holds with respect to ${ }_{2} X$. The same is true by considering a finite number of summands.

We have elsewhere shown in the form of a theorem that prevision choices are consumption choices. It follows that the notion of prevision of a risky asset has the same properties of the one of well-behaved preferences (see also Wold et al. (1952)). To say that wellbehaved preferences are monotonic, because more is better, and convex, because averages are weakly preferred to extremes, means that it turns out to be

$$
\mathbf{P}(X+Y)=\mathbf{P}(X)+\mathbf{P}(Y)
$$

as well as

$$
x^{1} \leq \mathbf{P}(X) \leq x^{m},
$$

where $\mathbf{P}$ stands for prevision or mathematical expectation of a random quantity viewed as a random gain. In general, given two random gains denoted by $X$ and $Y$, their possible values are expressed by $I(X)=\left\{x^{1}, x^{2}, \ldots, x^{m}\right\}$ and $I(Y)=\left\{y^{1}, y^{2}, \ldots, y^{m}\right\}$, with $x^{1}<x^{2}<\ldots<x^{m}$ and $y^{1}<y^{2}<\ldots<y^{m}$ without loss of generality. Additivity of $\mathbf{P}$ given by (5) and its convexity expressed by (6) are necessary and sufficient conditions for coherence. They are so fundamental that the whole theory of probability is based on them only. 
If we consider all possible evaluations of the probability of each state of nature of a contingent consumption plan being made by the consumer then they permit the choice of any value in the interval from 0 to 1 , endpoints included. They identify a convex set every time.

Given a portfolio consisting of two risky assets denoted by ${ }_{1} X$ and ${ }_{2} X$, the expected returns on the two risky assets jointly considered are obtained by decomposing a coherent prevision of a joint risky asset inside of a two-dimensional metric space. We denote it by $E^{2}$. They tell us how much the consumer is choosing to consume of one of the two risky assets and how much she is choosing to consume of the other, so they can be viewed as a consumption bundle belonging to a two-dimensional convex set (see also Ghirardato et al. (2005)). It is the budget set of the consumer established by the negative slope of the budget line coinciding with a hyperplane into $E^{2}$. Her budget set is also established by the two mutually orthogonal axes under consideration (see also Slovic et al. (1977)). The budget line is an equation of a linear function expressed in an implicit form whose variables are the two expected returns on the two risky assets into account. The prices of the prevision bundle viewed as a consumption bundle are conversely the two constants of such a linear function. On the other hand, it is not money alone that matters, but it is the consumption (to be understood as an average consumption within this context) that money can buy that is the ultimate good being chosen by the consumer (see also Drapeau and Kupper (2013)). It is evident that the budget constraint of the consumer requires that the amount of money spent on the two goods viewed as two risky assets be no more than the total amount she has to spend. Since it turns out to be

$$
c_{1}\left({ }_{1} X\right)+c_{2}\left({ }_{2} X\right) \leq c,
$$

the budget constraint is written as

$$
c_{1} \mathbf{P}\left({ }_{1} X\right)+c_{2} \mathbf{P}\left({ }_{2} X\right) \leq c,
$$

where $\left(c_{1}, c_{2}\right)$ are the prices of the two goods, whereas the amount of money the consumer has to spend is equal to $c$. We note that $c_{1}, c_{2}$ and $c$ are positive real numbers (Markowitz (1952)). It is clear that one has

$$
\mathbf{P}\left({ }_{1} X\right)={ }_{(1)} x^{1}{ }_{(1)} p_{1}+\ldots+{ }_{(1)} x^{m}{ }_{(1)} p_{m}
$$

and

$$
\mathbf{P}\left({ }_{2} X\right)={ }_{(2)} x^{1}{ }_{(2)} p_{1}+\ldots+{ }_{(2)} x^{m}{ }_{(2)} p_{m},
$$

with

$$
{ }_{(1)} p_{1}+\ldots+{ }_{(1)} p_{m}=1
$$

as well as

$$
{ }_{(2)} p_{1}+\ldots+{ }_{(2)} p_{m}=1 \text {. }
$$

The slope of the budget line expressed by

$$
c_{1} \mathbf{P}\left({ }_{1} X\right)+c_{2} \mathbf{P}\left({ }_{2} X\right)=c
$$

coincides with 


$$
-\frac{c_{1}}{c_{2}}
$$

Although the slope of the budget line expressed by (13) can change as the state of information and knowledge of the consumer changes, we note that it always passes through the point whose coordinates are given by

$$
\left(\sup I\left({ }_{1} Y\right), \sup I\left({ }_{2} Y\right)\right) \text {, }
$$

where we have $X={ }_{1} Y-{ }_{1} Z$ as well as ${ }_{2} X={ }_{2} Y-{ }_{2} Z$. It follows that all coherent expected returns on the portfolio consisting of two risky assets can be expressed by

$$
\frac{c_{1}}{c_{1}+c_{2}} \mathbf{P}\left({ }_{1} X\right)+\frac{c_{2}}{c_{1}+c_{2}} \mathbf{P}\left({ }_{2} X\right) \leq \frac{c}{c_{1}+c_{2}}
$$

whenever we use a linear and quadratic metric. On the other hand, the budget set of the consumer does not change whenever we multiply all prices and income by a positive number. It follows that the standard economic model of consumer behavior is even valid within this specific situation. The left-hand side of (16) is a weighted average of the two expected returns on the two risky assets under consideration. All coherent expected returns on the portfolio consisting of two risky assets give rise to a two-dimensional convex set. A joint distribution decomposed into two marginal distributions characterizes them (see also von Rosen (1989)). It is summarized by means of $\mathbf{P}\left({ }_{1} X_{2} X\right)$, where $\mathbf{P}\left({ }_{1} X_{2} X\right)$ is bilinear. After distinguishing finite sets given by $I\left({ }_{1} X\right), I\left({ }_{2} X\right)$ and $I\left({ }_{1} X{ }_{2} X\right)$ from uncountable sets coinciding with convex sets, we focus on two continuous goods denoted by $\mathbf{P}\left({ }_{1} X\right), \mathbf{P}\left({ }_{2} X\right)$ and obtained by using our geometric interpretation of conditions of coherence of $\mathbf{P}$. The consumer divides her monetary wealth given by

$$
\frac{c_{1}}{c_{1}+c_{2}}
$$

and

$$
\frac{c_{2}}{c_{1}+c_{2}}
$$

between the two risky assets, where it evidently turns out to be

$$
\frac{c_{1}}{c_{1}+c_{2}}+\frac{c_{2}}{c_{1}+c_{2}}=1
$$

Hence, we establish the following

Definition 2 After decomposing $\mathbf{P}\left({ }_{1} X_{2} X\right)$ inside of $E^{2}$, the consumer's demand functions that give the average consumption amounts of each of the two goods under consideration denoted by ${ }_{1} X$ and ${ }_{2} X$ (of which we are only interested in their previsions given by $\mathbf{P}\left({ }_{1} X\right)$ and $\left.\mathbf{P}\left({ }_{2} X\right)\right)$ are expressed by

$$
\mathbf{P}\left({ }_{1} X\right)=\left\{\mathbf{P}\left({ }_{1} X\right)\left[\left(c_{1}, c_{2}, c\right)\right]\right\}
$$

as well as 


$$
\mathbf{P}\left({ }_{2} X\right)=\left\{\mathbf{P}\left({ }_{2} X\right)\left[\left(c_{1}, c_{2}, c\right)\right]\right\},
$$

where $\mathbf{P}$ is always additive and convex as a consequence of its coherence.

Since $\mathbf{P}\left({ }_{1} X{ }_{2} X\right), \mathbf{P}\left({ }_{1} X\right)$ and $\mathbf{P}\left({ }_{2} X\right)$ belong to convex sets, it is evident that the quantities demanded denoted by $\mathbf{P}\left({ }_{1} X\right)$ and $\mathbf{P}\left({ }_{2} X\right)$ depend on the three elements identifying a two-dimensional convex set inside of which $\mathbf{P}\left({ }_{1} X_{2} X\right)$ is coherently decomposed into $\mathbf{P}\left({ }_{1} X\right)$ and $\mathbf{P}\left({ }_{2} X\right)$.

In this paper, we do not consider a joint distribution only. It follows that we innovatively define a stand-alone and double risky asset. We denote it by $X_{12}$. A portfolio consisting of two risky assets can then be viewed as a stand-alone and double risky asset. It is possible to consider $\mathbf{P}\left(X_{12}\right)$ whenever we use a non-linear and quadratic metric. We therefore define the expected return denoted by $\mathbf{P}\left(X_{12}\right)$ on the portfolio of the consumer who invests in two different risky assets (see also Davies and Satchell (2007)). We also define the variance of the portfolio return denoted by $\operatorname{Var}\left(X_{12}\right)$.

\section{Logical and probabilistic aspects concerning an ordered pair of contingent consumption plans}

Let $\mathcal{B}_{m}^{\perp}=\left\{\mathbf{e}_{i} \mid i \in I_{m}=\{1, \ldots, m\}\right\}$ be an orthonormal basis of $E^{m}$. Let $\left({ }_{1} X,{ }_{2} X\right)$ be an ordered pair of risky assets. We are then faced with two different partitions, where each of them is characterized by $m$ incompatible and exhaustive events (see also Battigalli and Siniscalchi (2003)). After considering $I\left({ }_{1} X\right)=\left\{{ }_{(1)} x^{1}, \ldots,{ }_{(1)} x^{m}\right\}$ and $I\left({ }_{2} X\right)=\left\{{ }_{(2)} x^{1}, \ldots,{ }_{(2)} x^{m}\right\}$ we establish the following

Definition 3 All states of nature of an ordered pair of contingent consumption plans jointly considered are obtained from the Cartesian product of the possible monetary values of two logically independent risky assets denoted by ${ }_{1} X$ and ${ }_{2} X$. Such risky assets give rise to a joint risky asset denoted by ${ }_{1} X_{2} X$. It is a function written in the form ${ }_{1} X{ }_{2} X: I\left({ }_{1} X\right) \times I\left({ }_{2} X\right) \rightarrow \mathbb{R}$, where it turns out to be ${ }_{1} X{ }_{2} X\left({ }_{(1)} x^{i},{ }_{(2)} x^{j}\right)={ }_{(1)} x^{i}{ }_{(2)} x^{j}$, with $i, j=1, \ldots, m$.

We are faced with an extension of a canonical expression for a random quantity. It is written in the form given by

$$
\begin{aligned}
{ }_{1} X{ }_{2} X= & \left.{ }_{(1)} x^{1}{ }_{(2)} x^{1}\right|_{(1)} E_{1}||_{(2)} E_{1}\left|+\ldots+{ }_{(1)} x^{i}{ }_{(2)} x^{j}\right|_{(1)} E_{i}||_{(2)} E_{j} \mid+\ldots \\
& +\left.{ }_{(1)} x^{m}{ }_{(2)} x^{m}\right|_{(1)} E_{m} \|_{(2)} E_{m} \mid,
\end{aligned}
$$

where it is possible to observe

$$
\left.\right|_{(1)} E_{i}||_{(2)} E_{j} \mid= \begin{cases}1, & \text { if }{ }_{(1)} E_{i} \text { and }{ }_{(2)} E_{j} \text { are both true } \\ 0, & \text { otherwise }\end{cases}
$$

for every $i, j=1, \ldots, m$.

We geometrically consider $_{(1)} \mathbf{x} \in E^{m}$ as well as ${ }_{(2)} \mathbf{x} \in E^{m}$. We write

$$
{ }_{(1)} \mathbf{x}={ }_{(1)} x^{i} \mathbf{e}_{i}
$$

and 


$$
{ }_{(2)} \mathbf{x}={ }_{(2)} x^{i} \mathbf{e}_{i}
$$

where it is evident that we use the Einstein summation convention. We note that ${ }_{(1)} \mathbf{x}$ and ${ }_{\text {(2) }} \mathbf{x}$ are linearly independent and uniquely represented with respect to $\mathcal{B}_{m}^{\perp}$ because there exists one and only one $m$-tuple of real numbers coinciding with the set $\left.{ }_{{ }_{(1)}} x^{i}\right\}$ and satisfying (24). There also exists one and only one $m$-tuple of real numbers coinciding with the set $\left\{{ }_{(2)} x^{i}\right\}$ and satisfying (25). We associate the contravariant components of ${ }_{(1)} \mathbf{x}$ and ${ }_{(2)} \mathbf{x}$ with the possible monetary values of ${ }_{1} X_{2} X$ expressed in the same unit of measurement. We note that ${ }_{1} X$ and ${ }_{2} X$ are two marginal risky assets with regard to ${ }_{1} X{ }_{2} X$ (de Finetti (1982b)).

Therefore, we observe that the possible monetary values of ${ }_{1} X_{2} X$ are geometrically expressed by an affine tensor of order 2 whose contravariant components coincide with ${ }_{(1)} x^{i}{ }_{(2)} x^{j}$, where we have $i, j=1, \ldots, m$. The covariant components of an affine tensor of order 2 represent the joint probabilities of the joint distribution of ${ }_{1} X$ and ${ }_{2} X$. It is possible to associate in an orderly manner the covariant components of an affine tensor of order 2 with the joint probabilities of the joint distribution of ${ }_{1} X$ and ${ }_{2} X$. Their number is overall equal to $m^{2}$. We write

$$
p=\left(p_{i j}\right)
$$

with $p \in E^{m} \otimes E^{m}$. We note that it turns out to be

$$
\sum_{i=1}^{m} \sum_{j=1}^{m} p_{i j}=1
$$

because all probabilistic evaluations being made by the consumer have to be coherent (de Finetti (1982a)).

\subsection{Metric aspects concerning an ordered pair of contingent consumption plans}

We say that an ordered pair of risky assets denoted by $\left({ }_{1} X,{ }_{2} X\right)$ is represented by an ordered triple of geometric entities denoted by

$$
\left({ }_{(1)} \mathbf{x},{ }_{(2)} \mathbf{x}, p_{i j}\right),
$$

with $(i, j) \in I_{m} \times I_{m}$.

We consider the notion of $\alpha$-product between ${ }_{(1)} \mathbf{x}$ and ${ }_{(2)} \mathbf{x}$ in order to establish a quadratic and linear metric on $E^{m}$. It is a scalar product obtained by using the joint probabilities of the joint distribution of ${ }_{1} X$ and ${ }_{2} X$ together with the contravariant components of ${ }_{(1)} \mathbf{x}$ and (2) $\mathbf{x}$. We then write

$$
\left\langle{ }_{(1)} \mathbf{x},{ }_{(2)} \mathbf{x}\right\rangle_{\alpha}={ }_{(1)} x^{i}{ }_{(2)} x^{j} p_{i j}={ }_{(1)} x^{i}{ }_{(2)} x_{i}
$$

where

$$
{ }_{(2)}{ }^{j} p_{i j}={ }_{(2)} x_{i}
$$

is a vector homography by means of which we evidently pass from ${ }_{(2)} x^{j}$ to ${ }_{(2)} x_{i}$ by using $p_{i j}$. This means that the mathematical expectation of a marginal risky asset is decomposed. On the other hand, if we decompose the mathematical expectation of the other marginal risky asset then we obtain the same result given by (29). Such a notion possesses all properties of 
the scalar product (with regard to (29), we use $\alpha$ as a subscript because all joint probabilities of the joint distribution of ${ }_{1} X$ and ${ }_{2} X$ are considered). In particular, if it turns out to be

$$
\left\langle{ }_{(1)} \mathbf{x},{ }_{(2)} \mathbf{x}\right\rangle_{\alpha}=0
$$

then ${ }_{(1)} \mathbf{x}$ and ${ }_{(2)} \mathbf{x}$ are $\alpha$-orthogonal.

We note that from the notion of $\alpha$-product it follows the one of $\alpha$-norm of an $m$-dimensional vector. We therefore write

$$
\left\|_{(1)} \mathbf{x}\right\|_{\alpha}^{2}=\left\langle{ }_{(1)} \mathbf{x},{ }_{(1)} \mathbf{x}\right\rangle_{\alpha}={ }_{(1)} x_{(1)}^{i} x^{i} p_{i i}
$$

as well as

$$
\left\|_{(2)} \mathbf{x}\right\|_{\alpha}^{2}=\left\langle{ }_{(2)} \mathbf{x},{ }_{(2)} \mathbf{x}\right\rangle_{\alpha}={ }_{(2)} x^{i}{ }_{(2)} x^{i} p_{i i}
$$

because the joint probabilities of the particular joint distributions under consideration whose covariant indices are not equal coincide with 0 .

Also, it is possible to show two metric inequalities. The former is given by

$$
\left|\left\langle_{(1)} \mathbf{x},{ }_{(2)} \mathbf{x}\right\rangle_{\alpha}\right| \leq\left\|_{(1)} \mathbf{x}\right\|_{\alpha}\left\|_{(2)} \mathbf{x}\right\|_{\alpha} .
$$

It is called the Schwarz's $\alpha$-generalized inequality. The latter is expressed by

$$
\left\|_{(1)} \mathbf{x}+{ }_{(2)} \mathbf{x}\right\|_{\alpha} \leq\left\|_{(1)} \mathbf{x}\right\|_{\alpha}+\left\|_{(2)} \mathbf{x}\right\|_{\alpha} .
$$

It is called the $\alpha$-triangle inequality.

From (34) it follows the notion of $\alpha$-cosine, so it is possible to write

$$
\cos \left(_{(1)} \mathbf{x},{ }_{(2)} \mathbf{x}\right)_{\alpha}=\frac{\left\langle_{(1)} \mathbf{x},{ }_{(2)} \mathbf{x}\right\rangle_{\alpha}}{\left\|_{(1)} \mathbf{x}\right\|_{\alpha}\left\|_{(2)} \mathbf{x}\right\|_{\alpha}} .
$$

What we have just said is valid whenever we consider changes of origin (see also Rockafellar et al. (2006)). Let ${ }_{X} d$ be a risky asset whose possible monetary values represent all deviations from a mean value. We denote it by $\overline{\mathbf{x}} \in E^{m}$, where it turns out to be

$$
\overline{\mathbf{x}}=\left(\begin{array}{c}
\bar{x}^{1}=\mathbf{P}(X) \\
\bar{x}^{2}=\mathbf{P}(X) \\
\vdots \\
\bar{x}^{m}=\mathbf{P}(X)
\end{array}\right) .
$$

We then say that ${ }_{X} d$ is geometrically identified with an $m$-dimensional vector of $E^{m}$ given by

$$
{ }_{\mathbf{x}} \mathbf{d}=\mathbf{x}-\overline{\mathbf{x}}
$$

Its contravariant components are expressed by

$$
{ }_{\mathbf{x}} d^{i}=x^{i}-\bar{x}^{i},
$$

with $i=1, \ldots, m$.

We write 


$$
{ }_{(2)} S^{(1)} \subset E^{m},
$$

where ${ }_{(2)} S^{(1)}$ is an $m$-dimensional linear space contained in $E^{m}$. If $X_{12}=\left\{{ }_{1} X,{ }_{2} X\right\}$ is a double risky asset then its marginal components denoted by ${ }_{1} X$ and ${ }_{2} X$ can geometrically be represented by two vectors of $E^{m}$ denoted by ${ }_{(1)} \mathbf{d}$ and ${ }_{(2)} \mathbf{x}$, where we have ${ }_{(1)} \mathbf{x},{ }_{(2)} \mathbf{x}, \mathbf{d} \in{ }_{(2)} S^{(1)}$. We write

$$
{ }_{(1)} \mathbf{d}={ }_{(1)} \mathbf{d}
$$

as well as

$$
{ }_{(2)} \mathbf{x}={ }_{(2)} \mathbf{d} .
$$

Given

$$
\mathbf{y}=\frac{c_{1}}{c_{1}+c_{2}}\left({ }_{1)} \mathbf{d}+\frac{c_{2}}{c_{1}+c_{2}}(2) \mathbf{d}\right.
$$

it is possible to obtain

$$
\begin{aligned}
\|\mathbf{y}\|_{\alpha}^{2}= & \left(\frac{c_{1}}{c_{1}+c_{2}}\right)^{2}\left\|_{(1)} \mathbf{d}\right\|_{\alpha}^{2}+2 \frac{c_{1}}{c_{1}+c_{2}} \frac{c_{2}}{c_{1}+c_{2}}\left\langle{ }_{(1)} \mathbf{d},{ }_{(2)} \mathbf{d}\right\rangle_{\alpha} \\
& +\left(\frac{c_{2}}{c_{1}+c_{2}}\right)^{2}\left\|_{(2)} \mathbf{d}\right\|_{\alpha}^{2},
\end{aligned}
$$

with

$$
\begin{aligned}
& \left\|_{(1)} \mathbf{d}\right\|_{\alpha}^{2}=\operatorname{Var}\left({ }_{1} X\right), \\
& \left\|_{(2)} \mathbf{d}\right\|_{\alpha}^{2}=\operatorname{Var}\left({ }_{2} X\right)
\end{aligned}
$$

as well as

$$
\left\langle{ }_{(1)} \mathbf{d},{ }_{(2)} \mathbf{d}\right\rangle_{\alpha}=\operatorname{Cov}\left({ }_{1} X,{ }_{2} X\right)
$$

We then establish the following

Definition 4 We call linear metric the expression given by (44). Since it is possible to write $\left.\left\|_{(1)} \mathbf{d}-{ }_{(2)} \mathbf{d}\right\|_{\alpha}^{2}=\left\|_{(1)} \mathbf{d}\right\|_{\alpha}^{2}+\left\|_{(2)} \mathbf{d}\right\|_{\alpha}^{2}-2 \zeta_{(1)} \mathbf{d},{ }_{(2)} \mathbf{d}\right\rangle_{\alpha}$, it evidently derives from the notion of $\alpha$-distance between two single risky assets of a portfolio consisting of two risky assets whose possible monetary values are subjected to two changes of origin.

We note the following

Remark 1 Whenever we consider the variance of the portfolio return by using a linear and quadratic metric we are faced with a joint distribution depending on the notion of ordered pair of risky assets. Its covariance is consequently calculated. 
If (44) holds then a portfolio consisting of two risky assets is not viewed as a standalone and double risky asset.

\section{Two contingent consumption plans jointly considered that are independent of the notion of ordered pair}

We note the following

Remark 2 Let ${ }_{1} X$ and ${ }_{2} X$ be two linearly independent risky assets identifying two contingent consumption plans. We suppose that each of them is characterized by $m$ possible monetary values. The possible monetary values of two risky assets separately considered are logically represented by the contravariant components of two $m$-dimensional vectors. The possible monetary values of two logically independent risky assets jointly considered have consequently to be represented by the contravariant components of a tensor of order 2 . It is an antisymmetric tensor of order 2 whenever we are interested in defining a stand-alone and double risky asset obtained by fusing together its marginal components denoted by ${ }_{1} X$ and ${ }_{2} X$.

We then pass from an ordered pair of contingent consumption plans to two contingent consumption plans which are jointly considered regardless of the notion of ordered pair. We have then to consider a double risky asset denoted by

$$
X_{12}=\left\{{ }_{1} X,{ }_{2} X\right\}
$$

whose possible monetary values coincide with the contravariant components of an antisymmetric tensor of order 2 . We say that $X_{12}$ is a portfolio of two risky assets jointly considered that are independent of the notion of ordered pair. Hence, after choosing $\mathrm{m}^{2}$ joint probabilities connected with ${ }_{1} X_{2} X$, we observe that it is necessary to consider four joint distributions characterizing ${ }_{1} X{ }_{1} X,{ }_{1} X{ }_{2} X,{ }_{2} X{ }_{1} X$ and ${ }_{2} X{ }_{2} X$, with

$$
\begin{aligned}
& { }_{1} X{ }_{1} X: I\left({ }_{1} X\right) \times I\left({ }_{1} X\right) \rightarrow \mathbb{R}, \\
& { }_{2} X{ }_{2} X: I\left({ }_{2} X\right) \times I\left({ }_{2} X\right) \rightarrow \mathbb{R}
\end{aligned}
$$

and

$$
{ }_{2} X{ }_{1} X: I\left({ }_{2} X\right) \times I\left({ }_{1} X\right) \rightarrow \mathbb{R},
$$

in order to release $X_{12}$ from the notion of ordered pair. We note that the marginal components of $X_{12}$ denoted by ${ }_{1} X$ and ${ }_{2} X$ are not put near unlike what happens when we jointly consider ${ }_{i} X$ and ${ }_{j} X$, where we have $i, j=1,2$. Each probability distribution of a marginal risky asset is viewed as a particular joint distribution. This implies that all off-diagonal joint probabilities of a two-way table, where the number of rows is equal to the one of columns, coincide with 0 .

We prove the following

Theorem 1 The mathematical expectation of $X_{12}=\left\{{ }_{1} X,{ }_{2} X\right\}$ coincides with the determinant of a square matrix of order 2. Each element of such a determinant is a real number coinciding with the mathematical expectation of ${ }_{i} X_{j} X$, where we have $i, j=1,2$. 
An affine tensor of order 2 representing the possible monetary values of ${ }_{1} X{ }_{2} X$, where ${ }_{1} X{ }_{2} X$ corresponds to $\left({ }_{1} X,{ }_{2} X\right)$, is written in the form

$$
T={ }_{(1)} \mathbf{x} \otimes{ }_{(2)} \mathbf{x}={ }_{(1)} x^{i}{ }_{(2)} x^{j} \mathbf{e}_{i} \otimes \mathbf{e}_{j} .
$$

An affine tensor of order 2 representing the possible monetary values of ${ }_{2} X{ }_{1} X$, where ${ }_{2} X{ }_{1} X$ corresponds to $\left({ }_{2} X,{ }_{1} X\right)$, is conversely written in the form

$$
T={ }_{(2)} \mathbf{x} \otimes{ }_{(1)} \mathbf{x}={ }_{(2)} x^{j}{ }_{(1)} x^{i} \mathbf{e}_{j} \otimes \mathbf{e}_{i} .
$$

We have written a same affine tensor of order 2 denoted by $T$ whose $m^{2}$ contravariant components are not the same. If we pass from (52) to (53) then we note that the contravariant components whose upper indices are equal do not change. If we pass from (52) to (53) then we note that the contravariant components whose upper indices are not equal change. It follows that we write an antisymmetric tensor of order 2 in the form

$$
T=\sum_{i<j}\left({ }_{(1)} x_{(2)}^{i} x^{j}-{ }_{(1)}{ }^{x^{j}}{ }_{(2)} x^{i}\right) \mathbf{e}_{i} \otimes \mathbf{e}_{j}
$$

because we have to consider (52) and (53) together. We have written $i<j$ under the summation symbol since it is easy to realize that if it turns out to be $i=j$ then every contravariant component inside parentheses is equal to 0 . Hence, we denote by ${ }_{12} x$ an antisymmetric tensor of order 2 logically identifying $X_{12}$. We write

$$
{ }_{12} x^{(i j)}=\left|\begin{array}{ll}
(1)^{x^{i}} & { }_{(1)} x^{j} \\
{ }_{(2)} x^{i} & { }_{(2)} x^{j}
\end{array}\right|={ }_{(1)} x^{i}{ }_{(2)} x^{j}-{ }_{(1)} x^{\left.x^{j}\right)} x^{i}
$$

in order to identify the strict contravariant components of it. We have $i<j$. The number of such components is overall equal to

$$
\left(\begin{array}{c}
m \\
2
\end{array}\right)
$$

The corresponding strict covariant components of ${ }_{12} x$ are given by

$$
{ }_{12} x_{(i j)}=\left|\begin{array}{ll}
{ }_{(1)} x_{i} & { }_{(1)} x_{j} \\
{ }_{(2)} x_{i} & \text { (2) } x_{j}
\end{array}\right|=\left|\begin{array}{ll}
{ }_{(1)} x^{j} p_{j i} & { }^{x} x^{i} p_{i j} \\
{ }_{(2)} x^{j} p_{j i} & \text { (2) } x^{i} p_{i j}
\end{array}\right|,
$$

where we have $i<j$. By taking all joint probabilities of the joint distribution of ${ }_{1} X$ and ${ }_{2} X$ into account, such covariant components are obtained by considering some probabilities every time. This means that we could be faced with several vector homographies. Given a two-way table containing all joint probabilities, we always consider those probabilities belonging to a row or column of it in order to obtain a covariant component of an $m$-dimensional vector. We do not compute the scalar value of (57). We have to note that the number of the strict contravariant and covariant components of ${ }_{12} x$ is absolutely unimportant. We always obtain the same outcome independently of such a number. We put together (55) and (57), where (55) and (57) contain all strict contravariant and covariant components of ${ }_{12} x$ at the same time. We always put together (55) and (57) in the same way. We always associate ${ }_{(1)} x^{i}$ with ${ }_{(1)} x_{i},{ }_{(1)} x^{j}$ with ${ }_{(2)} x_{j},{ }_{(2)} x^{i}$ with ${ }_{(1)} x_{i}$ and ${ }_{(2)} x^{j}$ with ${ }_{(2)} x_{j}$. After putting together (55) and (57), whose structure is evidently the one of two determinants because we are considering multilinear matters, we obtain different single terms (monomials). It follows 
that a variable index appearing twice in a monomial implies summation of it over all values of the index (hence, every time it is possible to obtain a polynomial by using the Einstein notation). On the other hand, all strict contravariant and covariant components of ${ }_{12} x$ are simultaneously identified with two determinants because, in general, the determinant of a square matrix is the most exemplary multilinear relationship as well as a linear combination of basis vectors is the most exemplary linear relationship. We obtain the mathematical expectation of $X_{12}$ given by

$$
\left\|_{12} x\right\|_{\alpha}^{2}=\left|\begin{array}{cc}
\left\|_{(1)} \mathbf{x}\right\|_{\alpha}^{2} & \left\langle{ }_{(1)} \mathbf{x},{ }_{(2)} \mathbf{x}\right\rangle_{\alpha} \\
\left\langle_{(2)} \mathbf{x},{ }_{(1)} \mathbf{x}\right\rangle_{\alpha} & \left\|_{(2)} \mathbf{x}\right\|_{\alpha}^{2}
\end{array}\right|=\left\|_{(1)} \mathbf{x}\right\|_{\alpha}^{2}\left\|_{(2)} \mathbf{x}\right\|_{\alpha}^{2}-\left(\left\langle_{(1)} \mathbf{x},{ }_{(2)} \mathbf{x}\right\rangle_{\alpha}\right)^{2},
$$

where we evidently observe

$$
\left\langle{ }_{(1)} \mathbf{x},{ }_{(2)} \mathbf{x}\right\rangle_{\alpha}=\left\langle{ }_{(2)} \mathbf{x},{ }_{(1)} \mathbf{x}\right\rangle_{\alpha} .
$$

By putting together (55) and (57) we are always faced with four joint distributions characterizing ${ }_{1} X{ }_{1} X,{ }_{1} X{ }_{2} X,{ }_{2} X{ }_{1} X$ and ${ }_{2} X{ }_{2} X$ that are all summarized. We write

$$
\left\|_{12} x\right\|_{\alpha}^{2}=\mathbf{P}\left(X_{12}\right)
$$

where it turns out to be

$$
\begin{aligned}
\mathbf{P}\left(X_{12}\right) & =\left|\begin{array}{cc}
\left\|_{(1)} \mathbf{x}\right\|_{\alpha}^{2} & \left\langle_{(1)} \mathbf{x},{ }_{(2)} \mathbf{x}\right\rangle_{\alpha} \\
\left\langle_{(2)} \mathbf{x},{ }_{(1)} \mathbf{x}\right\rangle_{\alpha} & \left\|_{(2)} \mathbf{x}\right\|_{\alpha}^{2}
\end{array}\right| \\
& =\left|\begin{array}{ll}
{ }_{(1)} x^{i}{ }_{(1)} x^{i} p_{i i}^{(11)}={ }_{(1)} x^{i}{ }_{(1)} x_{i} & { }_{(1)} x^{j}{ }_{(2)} x^{i} p_{i j}^{(12)}={ }_{(1)} x^{j}{ }_{(2)} x_{j} \\
{ }_{(2)} x^{i}{ }_{(1)} x^{j} p_{j i}^{(21)}={ }_{(2)} x^{i}{ }_{(1)} x_{i} & { }_{(2)}{ }^{x^{j}}{ }_{(2)} x^{x^{j}} p_{j j}^{(22)}={ }_{(2)} x_{(2)}{ }^{x_{j}} x_{j}
\end{array}\right| .
\end{aligned}
$$

We note that $p^{(11)}$ is the tensor of all joint probabilities associated with $\left(_{1} \mathbf{x},{ }_{1} \mathbf{x}\right)$. The same is true for all others contained in (61). It is possible to observe that in general it turns out to be

$$
\mathbf{P}\left({ }_{1} X_{2} X\right) \neq \mathbf{P}\left(X_{12}\right)
$$

We finally write

$$
\mathbf{P}\left(X_{12}\right)=\left|\begin{array}{ll}
\mathbf{P}\left({ }_{1} X_{1} X\right) & \mathbf{P}\left({ }_{1} X_{2} X\right) \\
\mathbf{P}\left({ }_{2} X_{1} X\right) & \mathbf{P}\left({ }_{2} X_{2} X\right)
\end{array}\right|
$$

where the determinant of the square matrix of order 2 under consideration is a bilinear function of the columns of it.

Given ${ }_{1} X$ and ${ }_{2} X$ and their coherent previsions denoted by $\mathbf{P}\left({ }_{1} X\right)$ and $\mathbf{P}\left({ }_{2} X\right)$, we consider all deviations from $\mathbf{P}\left({ }_{1} X\right)$ and $\mathbf{P}\left({ }_{2} X\right)$ of the possible monetary values of ${ }_{1} X$ and ${ }_{2} X$. We then prove the following

Theorem 2 The variance of $X_{12}=\left\{{ }_{1} X,{ }_{2} X\right\}$ coincides with the determinant of a square matrix of order 2. Each element of such a determinant is a real number coinciding with the variance of ${ }_{1} X$ and ${ }_{2} X$ and with their covariance.

All deviations from $\mathbf{P}\left({ }_{1} X\right)$ and $\mathbf{P}\left({ }_{2} X\right)$ of the possible monetary values of ${ }_{1} X$ and ${ }_{2} X$ are translations. It is then possible to write 


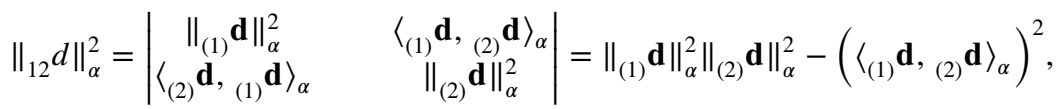

where ${ }_{12} d$ is an antisymmetric tensor of order 2 logically representing $X_{12}$. We are faced with changes of origin of the possible monetary values of ${ }_{1} X$ and ${ }_{2} X$. We write

$$
\left\|_{12} d\right\|_{\alpha}^{2}=\operatorname{Var}\left(X_{12}\right)=\sigma_{X_{12}}^{2} .
$$

We note that it turns out to be

$$
\left\langle{ }_{(1)} \mathbf{d},{ }_{(2)} \mathbf{d}\right\rangle_{\alpha}=\left\langle{ }_{(2)} \mathbf{d},{ }_{(1)} \mathbf{d}\right\rangle_{\alpha}=\operatorname{Cov}\left({ }_{1} X,{ }_{2} X\right)=\operatorname{Cov}\left({ }_{2} X,{ }_{1} X\right),
$$

so it is possible to write

$$
\operatorname{Var}\left(X_{12}\right)=\left|\begin{array}{cc}
\operatorname{Var}\left({ }_{1} X\right) & \operatorname{Cov}\left({ }_{1} X,{ }_{2} X\right) \\
\operatorname{Cov}\left({ }_{2} X,{ }_{1} X\right) & \operatorname{Var}\left({ }_{2} X\right)
\end{array}\right|
$$

If we are faced with the variance of $X_{12}$ then ${ }_{1} X$ and ${ }_{2} X$ are fused together. In general, if we compute only the covariance of ${ }_{1} X$ and ${ }_{2} X$ (in addition to the variance of each of them) then they are simply put near.

We observe that $\mathbf{P}\left(X_{12}\right)$ is coherent in the same way as $\mathbf{P}\left({ }_{1} X\right), \quad \mathbf{P}\left({ }_{2} X\right)$ and $\mathbf{P}\left({ }_{1} X_{2} X\right)=\mathbf{P}\left({ }_{2} X{ }_{1} X\right)$, where $\mathbf{P}\left({ }_{1} X\right)$ and $\mathbf{P}\left({ }_{2} X\right)$ are both linear indices (de Finetti (1989)).

We note the following

Remark 3 The origin of the variability of $X_{12}$ is not standardized, but it depends on the state of information and knowledge of the consumer. All deviations from $\mathbf{P}\left({ }_{1} X\right)$ and $\mathbf{P}\left({ }_{2} X\right)$ of the possible monetary values of ${ }_{1} X$ and ${ }_{2} X$ evidently depend on the state of information and knowledge of the consumer.

We establish the following

Definition 5 We call non-linear metric the expression given by (64). It is the area of a 2-parallelepiped whose edges are two single risky assets having their possible monetary values that are subjected to two changes of origin. The strict components of ${ }_{12} d$ are the coordinates of such edges denoted by ${ }_{(1)} \mathbf{d}$ and ${ }_{(2)} \mathbf{d}$.

We note the following

Remark 4 A non-linear metric given by (64) is invariant with regard to two different translations concerning ${ }_{(1)} \mathbf{d}$ and ${ }_{(2)} \mathbf{d}$ obtained by using two arbitrary vectors of $E^{m}$. It is also invariant with regard to two different rotations concerning ${ }_{(1)} \mathbf{d}$ and ${ }_{(2)} \mathbf{d}$ obtained by using two different $m \times m$ orthogonal matrices.

\section{Discussion and conclusion}

In this paper, the objects of consumer choice are probability distributions. They have therefore to be summarized. It follows that we define two consumer's demand functions connected with two contingent consumption plans. They are based on the notion of prevision 
of a random quantity. Since the possible values of a random quantity are of a monetary nature, the notion of prevision of a random quantity coincides with the one of price. In general, among those decisions leading to different random gains, the best choice being made by the consumer must then be the one that leads to the random gain with the highest price.

We have shown that coherent prevision choices are consumption choices, where the latter have to be meant as average consumption choices within this context. We have firstly considered all coherent summary measures of a joint distribution because we refer ourselves to the standard economic model of consumer behavior. The consumer chooses one of them in the same way as she chooses a consumption bundle. Given a joint distribution, it is secondly possible to consider four joint distributions in order to define a stand-alone and double random quantity. Their marginal distributions never change with regard to the ones of the starting joint distribution.

In this paper, our results are based on a particular approach to the origin of the variability of probability distributions. It is not standardized, but it always depends on the state of information and knowledge of the consumer. On the other hand, such a state can continuously be enriched by the flow of new information (see also Jurado et al. (2015)). Moreover, those results that are gradually learned or observed with regard to more or less analogous situations and cases enrich it as well (see also Korsakienè et al. (2019)).

It is possible to study prevision choices by means of revealed preference theory as well (see also Chambers et al. (2017)). It is known that the idea of revealed preference has an operational nature in the same way as the notion of prevision and utility (see also Halevy et al. (2018)). Both a theoretical extension of revealed preference theory and its empirical applications based on experimental data can be introduced (see also Echenique (2020)). How the consumer maximizes her utility associated with prevision bundles can consequently be studied. It is possible to study issues of correlation, variability, dissimilarity, linear regression and so on by using different summary measures of probability distributions inside of a linear space over $\mathbb{R}$. Such measures can be obtained by using both a linear metric and a non-linear one. In particular, the latter can innovatively be used in order to obtain an extension of the mean-variance model as well. If we consider more than two probability distributions then all prevision and riskiness choices being made by the consumer can be ranked inside of a linear space over $\mathbb{R}$ (see also Johnson and Payne (1985)).

A probability distribution is completely characterized by its mathematical expectation and variance, where the variance is a measure of the riskiness of the wealth distribution under consideration. In particular, both mathematical expectation and variance of $X_{12}$ have been obtained by means of the notion of $\alpha$-norm of an antisymmetric tensor of order 2 . Thus, they always exist within this context. If the consumer is risk averse then a higher expected return denoted by $\mathbf{P}\left(X_{12}\right)$ makes her better off and a higher variance denoted by $\operatorname{Var}\left(X_{12}\right)$ makes her worse off, where $\mathbf{P}\left(X_{12}\right)$ and $\operatorname{Var}\left(X_{12}\right)$ are evidently two average values characterizing her consumption choices.

Funding Open access funding provided by Università degli Studi della Campania Luigi Vanvitelli within the CRUI-CARE Agreement.

Open Access This article is licensed under a Creative Commons Attribution 4.0 International License, which permits use, sharing, adaptation, distribution and reproduction in any medium or format, as long as you give appropriate credit to the original author(s) and the source, provide a link to the Creative Commons licence, and indicate if changes were made. The images or other third party material in this article are included in the article's Creative Commons licence, unless indicated otherwise in a credit line to the material. If material is not included in the article's Creative Commons licence and your intended use is not 
permitted by statutory regulation or exceeds the permitted use, you will need to obtain permission directly from the copyright holder. To view a copy of this licence, visit http://creativecommons.org/licenses/by/4.0/.

\section{References}

Angelini, P., Maturo, F.: Non-parametric probability distributions embedded inside of a linear space provided with a quadratic metric. Mathematics 8(11), 1901 (2020)

Anscombe, F.J., Aumann, R.J.: A definition of subjective probability. Ann. Math. Stat. 34(1), 199-205 (1963)

Battigalli, P., Siniscalchi, M.: Rationalization and incomplete information. Adv. Theor. Econ. 3(1). (2003). https://doi.org/10.2202/1534-5963.1073

Berti, P., Rigo, P.: On coherent conditional probabilities and disintegrations. Ann. Math. Artif. Intell. 35(1), 71-82 (2002)

Berti, P., Regazzini, E., Rigo, P.: Strong previsions of random elements. Stat. Methods Appl. (J. Italian Stat. Soc.) 10(1), 11-28 (2001)

Capotorti, A., Coletti, G., Vantaggi, B.: Standard and nonstandard representability of positive uncertainty orderings. Kybernetika 50(2), 189-215 (2014)

Cassese, G., Rigo, P., Vantaggi, B.: A special issue on the mathematics of subjective probability. Decis. Econ. Finance 43(1), 1-2 (2020)

Chambers, C.P., Echenique, F., Shmaya, E.: General revealed preference theory. Theo. Econ. 12(2), 493511 (2017)

Coletti, G., Petturiti, D., Vantaggi, B.: When upper conditional probabilities are conditional possibility measures. Fuzzy Sets Syst. 304, 45-64 (2016)

Davies, G.B., Satchell, S.E.: The behavioural components of risk aversion. J. Math. Psychol. 51(1), 1-13 (2007)

Dinçer, H., Hošková-Mayerová, Š, Korsakienė, R., Yüksel, S.: IT2-based multidimensional evaluation approach to the signaling: investors' priorities for the emerging industries. Soft Comput. 24(18), 13517-13534 (2020)

Drapeau, S., Kupper, M.: Risk preferences and their robust representation. Math. Operations Res. 38(1), 28-62 (2013)

de Finetti, B.: The role of Dutch Books and of proper scoring rules. Br. J. Psychol. Sci. 32, 55-56 (1981)

de Finetti, B.: Probability: the different views and terminologies in a critical analysis. In: Cohen, L.J., Łoś, J., Pfeiffer, H., Podewski, K.P. (eds.) Logic, pp. 391-394. North-Holland Publishing Company, Amsterdam, Methodology and Philosophy of Science VI (1982a)

Echenique, F.: New developments in revealed preference theory: decisions under risk, uncertainty, and intertemporal choice. Ann. Rev. Econ. 12(1), 299-316 (2020)

de Finetti, B.: The proper approach to probability. In: Koch, G., Spizzichino, F. (eds.) Exchangeability in Probability and Statistics, pp. 1-6. North-Holland Publishing Company, Amsterdam (1982b)

de Finetti, B.: Probabilism: a critical essay on the theory of probability and on the value of science. Erkenntnis 31(2-3), 169-223 (1989)

Ghirardato, P., Maccheroni, F., Marinacci, M.: Certainty independence and the separation of utility and beliefs. J. Econ. Theo. 120(1), 129-136 (2005)

Gilio, A., Sanfilippo, G.: Conditional random quantities and compounds of conditionals. Studia logica 102(4), 709-729 (2014)

Halevy, Y., Persitz, D., Zrill, L.: Parametric recoverability of preferences. J. Political Econ. 126(4), 15581593 (2018)

Hoskova-Mayerova, S., Maturo, A.: Decision-Making Process using Hyperstructures and Fuzzy Structures in Social Sciences, pp. 103-111. Springer International Publishing, Cham (2018)

Hošková-Mayerová, Š, Maturo, F., Kacprzyk, J.: Mathematical-Statistical Models and Qualitative theories for economic and social sciences, vol. 104. Springer, Berlin (2017)

Johnson, E.J., Payne, J.W.: Effort and accuracy in choice. Manag. Sci. 31(4), 395-414 (1985)

Jurado, K., Ludvigson, S.C., Ng, S.: Measuring uncertainty. Am. Econ. Rev. 105(3), 1177-1216 (2015)

Korsakienė, R., Bekešienè, S.: Hošková-Mayerová, Š: The effects of entrepreneurs' characteristics on internationalisation of gazelle firms: a case of Lithuania. Econ. Res.-Ekonomska Istraživanja 32(1), 2864 2881 (2019)

Machina, M.J.: Choice under uncertainty: problems solved and unsolved. J. Econ. Perspect. 1(1), 121-154 (1987) 
Markowitz, H.: The utility of wealth. J. Political Econ. 60(2), 151-158 (1952)

Maturo, F.: On an extension of the Dubins conditional probability axiomatic to coherent probability of fuzzy events. Italian J. Pure Appl. Math. 2018(39), 810-821 (2018a)

Maturo, F.: A subjective approach to fuzzy events and their coherent probability assessment for decision making under uncertainty. J. Interdiscip. Math. 21(6), 1427-1445 (2018b). https://doi.org/10.1080/ 09720502.2018.1456006

Nunke, R.J., Savage, L.J.: On the set of values of a nonatomic, finitely additive, finite measure. Proc. Am. Math. Soc. 3(2), 217-218 (1952)

Pfanzagl, J.: Subjective probability derived from the Morgenstern-von Neumann utility theory. In: Shubik, M. (ed.) Essays in Mathematical Economics in Honor of Oskar Morgenstern, pp. 237-251. Princeton University Press, Princeton (1967)

Pompilj, G.: On intrinsic independence. Bull. Int. Stat. Inst. 35(2), 91-97 (1957)

Regazzini, E.: Finitely additive conditional probabilities. Rendiconti del Seminario Matematico e Fisico di Milano 55(1), 69-89 (1985)

Rockafellar, R.T., Uryasev, S., Zabarankin, M.: Generalized deviations in risk analysis. Finance Stoch. 10, 51-74 (2006)

Schmeidler, D.: Subjective probability and expected utility without additivity. Econometrica 57(3), 571-587 (1989)

Slovic, P., Fischhoff, B., Lichtenstein, S.: Behavioral decision theory. Ann. Rev. Psychol. 28, 1-39 (1977)

von Neumann, J.: Examples of continuous geometries. Proc. Natl. Acad. Sci. United States of Am. 22(2), 101-108 (1936)

von Rosen, D.: Maximum likelihood estimators in multivariate linear normal models. J. Multivar. Anal. 31(2), 187-200 (1989)

Viscusi, W.K., Evans, W.N.: Behavioral probabilities. J. Risk Uncertain. 32(1), 5-15 (2006)

Wold, H., Shackle, G.L.S., Savage, L.J.: Ordinal preferences or cardinal utility? Econometrica 20(4), 661664 (1952)

Publisher's Note Springer Nature remains neutral with regard to jurisdictional claims in published maps and institutional affiliations. 